\title{
Second-harmonic generation in graded metallic films*
}

\author{
J. P. Huang\#, K. W. Yu \\ Department of Physics, The Chinese University of Hong Kong, Shatin, NT, Hong Kong
}

\begin{abstract}
We study the effective second-harmonic generation (SHG) susceptibility in graded metallic films by invoking the local field effects exactly, and further numerically demonstrate that the graded metallic films can serve as a novel optical material for producing a broad structure in both the linear and SHG response and an enhancement in the SHG signal.
\end{abstract}

ocis: $160.4330,310.6860,160.4670,160.4760$.

${ }^{*}$ This paper has appeard in Optics Letters 30, 275 (2005). 
There are much recent interest and practical need for nonlinear optical materials that process large nonlinear susceptibility or optimal figure of merit. A large enhancement in nonlinear responses has been found for a sub-wavelength multilayer (i.e., thin film) of titanium dioxide and conjugated polymer [1]. For nonlinear effects other than the Kerr effect, Hui et al. [2] derived general expressions for the effective susceptibility for the second-harmonic generation (SHG) in a binary composite of random dielectrics. They have also studied the thickness dependence of effective SHG susceptibility in films of random dielectrics and in composites with coated small particles [3,4]. Graded materials with various functionalities appear in nature and in fabricated materials. Graded thin films have many applications as the gradation profile provides an additional control on the physical properties. Graded thin films often possess different optical properties [5], when compared to bulk materials. It is known that graded materials have quite different physical properties from the homogeneous materials [6]. Also, it has been observed that the compositionally graded barium strontium titanate thin films have better electric properties than a single-layer barium strontium titanate film with the same compositions [7]. How to achieve enhanced SHG is up to now a challenging agenda $[8,9]$. In this paper, we shall investigate SHG in a graded metallic film with an intrinsic SHG response and a graded linear response in the metallic dielectric function.

Let us consider a graded metallic film with thickness $L$, with the gradation profile in the direction perpendicular to the film. If we only include quadratic nonlinearities, the local constitutive relation between the displacement field $\mathbf{D}(z)$ and the electric field $\mathbf{E}(z)$ in the static case would be $D_{i}(z)=\sum_{j} \epsilon_{i j}(z) E_{j}(z)+\sum_{j k} \chi_{i j k}(z) E_{j}(z) E_{k}(z)[3,4]$ with $i=x, y, z$, where $D_{i}(z)$ and $E_{i}(z)$ are the $i$ th component of $\mathbf{D}(z)$ and $\mathbf{E}(z)$, respectively, and $\chi_{i j k}$ is the SHG susceptibility. Here $\epsilon_{i j}(z)$ denotes the linear dielectric function, which we assume for simplicity to be isotropic $\epsilon_{i j}(z)=\epsilon(z) \delta_{i j}$. Both $\epsilon(z)$ and $\chi_{i j k}(z)$ are functions of $z$ and describe the gradation profiles. In general, when a monochromatic external field is applied, the nonlinearity will generate local potentials and fields at all harmonic frequency. For a finite frequency external electric field of the form $E_{0}=E_{0}(\omega) e^{-i \omega t}+$ c.c., the effective SHG 
susceptibility $\bar{\chi}_{2 \omega}$ can be extracted by considering the volume average of the displacement field at the frequency $2 \omega$ in the inhomogeneous medium [2-4]. Next, we adopt a graded dielectric profile that follows the Drude form

$$
\epsilon(z, \omega)=1-\frac{\omega_{p}^{2}(z)}{\omega(\omega+i \gamma(z))},
$$

where $0 \leq z \leq L$. The general form in Eq. (1) allows for the possibility of a gradation profile in the plasma frequency [e.g., Eq. (4)] and the relaxation rate [e.g., Eq. (5)]. For a $z$-dependent profile, we can make use of the equivalent capacitance for capacitors in series to evaluate the effective perpendicular linear response for a given frequency, $1 / \bar{\epsilon}(\omega)=(1 / L) \int_{0}^{L} \mathrm{~d} z[1 / \epsilon(z, \omega)]$. Using the continuity of the electrical displacement field, the local electric fields $E(z, \omega)$ satisfies

$$
\epsilon(z, \omega) E(z, \omega)=\bar{\epsilon}(\omega) E_{0}(\omega)
$$

where $E_{0}(\omega)$ is the applied field along $z$ axis. A $z$-dependent profile for the plasma frequency and the relaxation time can be achieved experimentally. One possible way may be to impose a temperature profile, as it has been observed that surface enhanced Raman scattering is sensitive to temperature [10]. Thus, one may tune the surface plasmon frequency by imposing an appropriate temperature gradient [11]. A temperature gradient may also be used in materials with a small band gap or with a profile on dopant concentrations. In this case, one may impose a charge carrier concentration profile to a certain extent. This effect, when coupled with materials with a significant intrinsic nonlinear susceptibility, will give us with a way to control the effective nonlinear response. For less conducting materials, one may replace the Drude form of dielectric constants by a Lorentz oscillator form. It may also be possible to fabricate dirty metal films in which the degree of disorder varies in the $z$-direction and hence leads to a relaxation-rate gradation profile.

The calculation of the effective nonlinear optical response then proceed by applying the expressions derived in Refs. [2,3]. Next, the effective SHG susceptibility $\bar{\chi}_{2 \omega}$ is given by $\bar{\chi}_{2 \omega}=\left\langle\chi_{2 \omega}(z) E_{\operatorname{lin}}(z, 2 \omega) E_{\operatorname{lin}}(z, \omega)^{2}\right\rangle /\left[E_{0}(2 \omega) E_{0}(\omega)^{2}\right]$, where $E_{\text {lin }}$ is the linear local electric 
field in the graded film with the same gradation profile but with a vanishing nonlinear response at the frequency concerned. Using Eq. (2) for the linear local fields, the effective SHG susceptibility can be rewritten as an integral over the film as

$$
\bar{\chi}_{2 \omega}=\frac{1}{L} \int_{0}^{L} \mathrm{~d} z \chi_{2 \omega}(z)\left(\frac{\bar{\epsilon}(2 \omega)}{\epsilon(z, 2 \omega)}\right)\left(\frac{\bar{\epsilon}(\omega)}{\epsilon(z, \omega)}\right)^{2} .
$$

To illustrate the SHG in graded films, we consider as a model system that the intrinsic SHG susceptibility $\chi_{2 \omega}(z)=\chi_{1}$ to be a real and positive frequency-independent constant and does not have a gradation profile. In doing so, we are allowed to focus on the enhancement of the SHG response when compared to $\chi_{1}$. To show the effects of gradation, here we take as a model plasma-frequency gradation profile

$$
\omega_{p}(z)=\omega_{p}(0)\left(1-C_{\omega} \cdot z\right),
$$

and a model relaxation-rate gradation profile [12]

$$
\gamma(z)=\gamma(\infty)+C_{\gamma} / z,
$$

where $C_{\omega}$ and $C_{\gamma}$ are constant parameters tuning the profile which is assumed to be linear in $z$. Here $\gamma(\infty)$ denotes the bulk damping coefficient, i.e., for $z \rightarrow \infty$. Set thickness $L=1$ so that we could focus on the film with a fixed thickness. Regarding the thickness dependence, we refer the reader to the work by Hui et al. [3]

Figure 1 shows the real and imaginary parts of the effective linear dielectric constant [Fig. 1(a) and Fig. 1(b)], and the real and imaginary parts of the effective SHG susceptibility [Fig. 1(c) and Fig. 1(d)] as a function of frequency $\omega / \omega_{p}(0)$. Also shown is the modulus of $\bar{\chi}_{2 \omega} / \chi_{1}$, see Fig. 1(e). The dielectric function gradation profile is given in Eqs. (1), (4) and (5) with $C_{\gamma}=0$, i.e., only a graded plasmon frequency is included. Throughout the calculations, the real part of the (linear) dielectric constant is negative naturally. In this case, a broad resonant plasmon band is observed. Note that for $C_{\omega} \rightarrow 0, \omega_{p}(z) / \omega_{p}(0) \rightarrow 1$. As $C_{\omega}$ increases, $\omega_{p}(z)$ takes on values within a broader range across the film, and leads to a broad plasmon band. Increasing $C_{\omega}$ also causes the plasmon peak to shift to lower frequencies. 
The reason is that, in analogous to capacitors in series, the effective dielectric constant of the film is dominated by the layer with the smallest dielectric constant. For the SHG response, the frequency dependence is quite complicated. As $C_{\omega}$ increases, it is noted that structures in the SHG response also shifts to the lower frequencies and the range of values of the SHG susceptibility increases as well. Figure 2 displays the results of model calculations in which a gradation profile of the relaxation rate of the form in Eq. (5) is also included. The effects are similar to those in Fig. 1. The SHG response is found to be enhanced more strongly in the presence of both a relaxation-rate gradation and a plasma-frequency gradation (see Fig. 1) than for plasmon-frequency gradation alone, especially at low frequencies. As $C_{\gamma}$ increases, the structures in the linear and SHG response both show a shift to lower frequencies. In Figs. 1-2, the quantities which can be both positive and negative are plotted in logarithm of modulus. When the quantities pass through zero, the logarithm will be very large, thus yielding spikes. In addition, we have used the normalized numbers in order to describe a general origin of the SHG in metal films rather than a specified metal film.

The point for achieving the present results is that one needs a sufficiently large gradient rather than a crucially particular form of the dielectric function or gradation profiles. Thus, it is expected that an enhancement in SHG responses will also be found in compositionally graded metal-dielectric composite films in which the fraction of metal component varies perpendicular to the film. In the present work, due to the symmetry of the film, we have only enhancement for the polarization perpendicular to the film (i.e., parallel to the direction of the gradient). In this polarization, the tangential component of electric field $E$ vanishes identically. Thus, the continuity of normal component of $D$ [see Eq. (2)] gives rise to the enhanced SHG. However, for the polarization parallel to the film, i.e., the tangential component does not vanish. In this polarization, it is the continuity of the tangential component of $E$ that leads to no enhancement at all [1].

Acknowledgments. We thank Professor Pak-Ming Hui for fruitful discussions and suggestions, as well as a critical reading of the manuscript. This work was supported by the Research Grants Council of the Hong Kong SAR Government. J. P. Huang's e-mail address 
is jphuang@mpip-mainz.mpg.de.

\#Present address: Max Planck Institute for Polymer Research, Mainz, Germany. 


\section{REFERENCES}

[1] G.L. Fischer, R.W. Boyd, R.J. Gehr, S.A. Jenekhe, J.A. Osaheni, J.E. Sipe, and L.A. Weller-Brophy, Phys. Rev. Lett. 74, 1871 (1995).

[2] P.M. Hui and D. Stroud, J. Appl. Phys. 82, 4740 (1997).

[3] P.M. Hui, C. Xu, and D. Stroud, Phys. Rev. B 69, 014202 (2004).

[4] P.M. Hui, C. Xu, and D. Stroud, Phys. Rev. B 69, 014203 (2004).

[5] H. Grüll, A. Schreyer, N.F. Berk, C.F. Majkrzak, and C.C. Han, Europhys. Lett. 50, $107(2000)$.

[6] G.W. Milton, The Theory of Composites (Cambrige University Press, Cambridge, 2002).

[7] S.G. Lu, X.H. Zhu, C.L. Mak, K.H. Wong, H.L.W. Chan, and C.L. Choy, Appl. Phys. Lett. 82, 2877 (2003).

[8] D. Pezzetta, C. Sibilia, M. Bertolotti, R. Ramponi, R. Osellame, M. Marangoni, J.W. Haus, M. Scalora, M.J. Bloemer, and C.M. Bowden, J. Opt. Soc. Am. B 19, 2102 (2002).

[9] G. Purvinis, P.S. Priambodo, M. Pomerantz, M. Zhou, T.A. Maldonado, and R. Magnusson, Opt. Lett. 29, 1108 (2004).

[10] B. Pettinger, X. Bao, I.C. Wilcock, M. Muhler, and G. Ertl, Phys. Rev. Lett. 72, 1561 (1994).

[11] H.-P. Chiang, P.T. Leung, and W.S. Tse, J. Phys. Chem. B 104, 2348 (2000).

[12] A.E. Neeves and M.H. Birnboim, J. Opt. Soc. Am. B 6, 787 (1989). 


\section{FIGURES}

FIG. 1. (a) $\operatorname{Re}[\bar{\epsilon}(\omega)]$, (b) $\operatorname{Im}[\bar{\epsilon}(\omega)]$ (linear optical absorption), (c) $\operatorname{Re}\left[\bar{\chi}_{2 \omega} / \chi_{1}\right]$, (d) $\operatorname{Im}\left[\bar{\chi}_{2 \omega} / \chi_{1}\right]$, and (e) Modulus of $\bar{\chi}_{2 \omega} / \chi_{1}$ versus the normalized incident angular frequency $\omega / \omega_{p}(0)$ for the dielectric function gradation profile [Eq. (1)] with various plasma-frequency gradation profile [Eq. (4)] and relaxation-rate gradation profile $[$ Eq. (5)]. Here $|\cdots|$ denotes the absolute value or modulus of $\cdots$. Parameters: $\gamma(\infty)=0.02 \omega_{p}(0)$ and $C_{\gamma}=0.0$.

FIG. 2. Same as Fig.1. Parameters: $\gamma(\infty)=0.02 \omega_{p}(0)$ and $C_{\omega}=0.6$. 


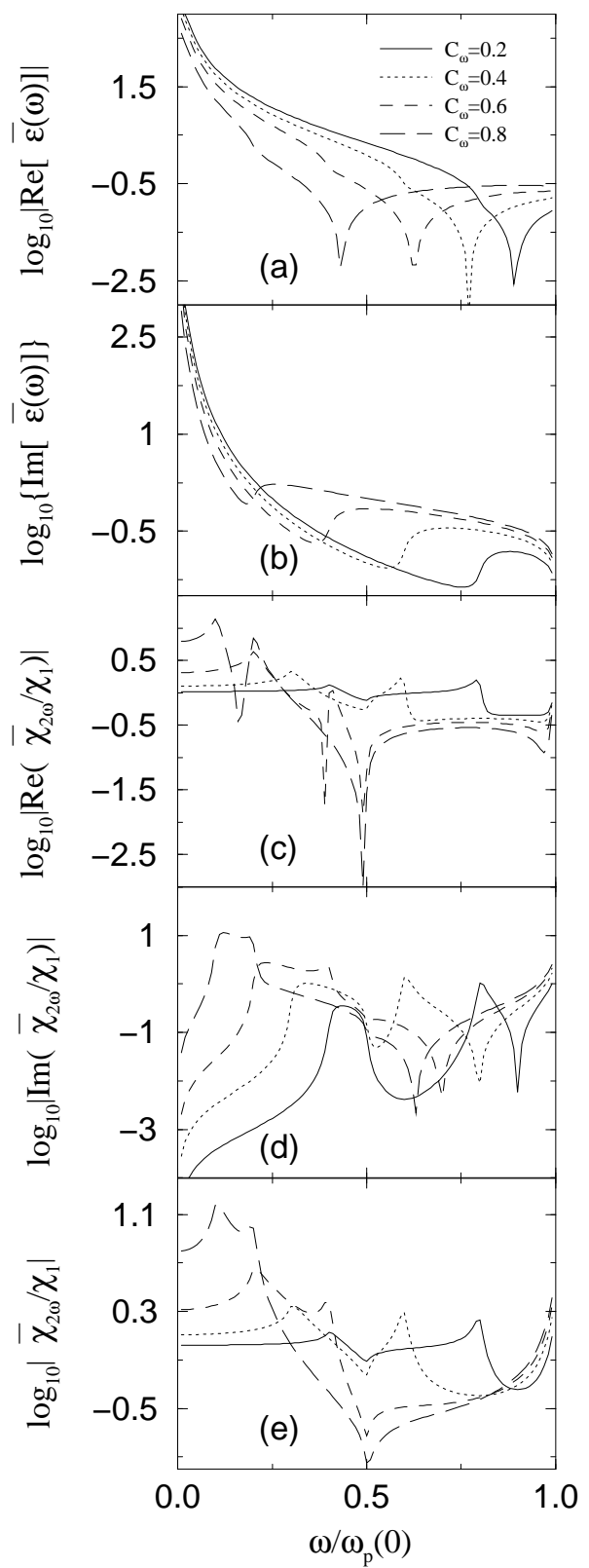

Fig. 1/Huang and $\mathrm{Yu}$ 


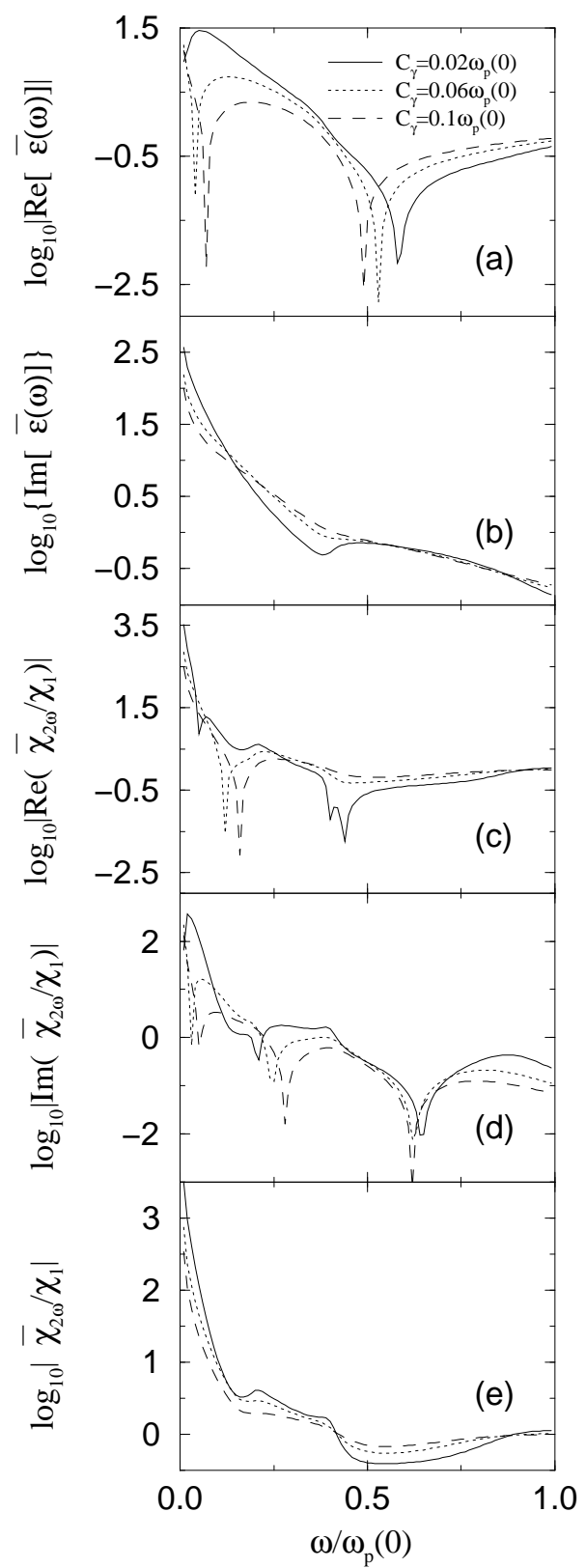

Fig. 2/Huang and $\mathrm{Yu}$ 